\title{
The Life Quality and Behavioral Stereotypes Concerning the Health of Students of Medical Professions
}

\author{
Kirill Kosilov ${ }^{1}{ }^{*}$ Ekaterina Fedorishcheva ${ }^{2}$, Olga Barabash ${ }^{3}$, Irina Gareeva ${ }^{4}$ \\ ${ }^{1}$ Far Eastern Federal University, Russia \\ ${ }^{2}$ Pacific State Medical University, Russia \\ ${ }^{3}$ Vladivostok State University of Economics and Service, Russia \\ ${ }^{4}$ Pacific State University, Russia \\ *Email: oton2000@mail.ru
}

\begin{abstract}
The goal of research consists in the study of correlation between the self-assessment of the life quality and behavioral stereotypes concerning the health of students of medical professions in terms of the structural and functional approach. 652 students of both sexes took part in this study, the sample showed the equal gender and age representation. Within the context of the empirical part of the study, the "MOS SF-36" questionnaire was used for studying the self-assessment of the life quality, for studying the basic behavioral stereotypes concerning the health, the questionnaire of the behavioral self-assessment of Pozdeeva (2008) as supplemented by authors was used. Correlation of variables was determined with a view to the Spearman's rank correlation coefficient. It was found that students assess their life quality within reference values; there was noted the high degree of implementation of behavioral attitudes concerning the health and psychohygiene; typically friends and teaching officers are referred to as behavioral influences while the variables quantitatively describing the life quality greatly correlate to indices characterizing the correlation with significant others, medical and physical activity of students.

The data obtained during investigation allowed us to suppose that the structural and functional approach provides means for effective analysis of correlation between the life quality and the processes of students` behavior adjustment to the educational system of the medical university. In accordance with the results obtained, there is observed the significant functional dependence between the processes of implementation of behavioral attitudes concerning the health-preserving behavior, medical, physical activity and the life quality self-assessment. In addition, the revealed inclination of students to imitation of health-preserving behavioral elements is in good agreement with theoretical concepts of $\mathrm{G}$. Tarde on imitating the behavior of significant others during the second insocialization, A. Bandura on "observation, imitation and identification" and is consistent with the principle of E. Thorndike concerning the choice of the most adaptive model of behavior in the new social environment.
\end{abstract}

Keywords: Health related quality of life, Medical students, Health behavior, Medical education.

\section{INTRODUCTION}

Formation of responsibilities and skills of future doctors has a number of essential features. In particular, every student spends the major part of study time in specialized classrooms equipped with diagnostic, laboratory, therapeutic and surgical equipment, breadboard models, simulators, in higher training becomes proficient straight at the patient's side under the guidance of the experienced doctor-teacher. Medical education is characterized by the tight schedule of training curriculum, intensive teaching loads, high and sometimes excessive loads on cognitive sphere, psychoemotional state as a whole [1-3]. 
The efficiency of the medical university educational system consists of a lot of different components, including academic teaching staff qualification, innovative teaching methods, material and technical possibilities of the university, balanced study schedule, the availability of ultimate nutrition, medical care, sports and recreation centers and so on [4]. One of the most essential factors associated with the teaching process efficiency is considered to be the students` health. The problems associated with the state of health and the high incidence of disease of students are widely covered in the native and foreign scientific literature $[5,6]$. At the same time, much less attention is given to the students` selfassessment of their life quality associated with health $[7,8]$.

The concept that implemented behavioral stereotypes and guide examples in relation to health directly impact on its objective findings has no need of additional evidences $[9,10]$. At the same time, the possible relationship between students' self-assessment of physical and mental status and implemented behavior directly affecting the objective indicators of health, remains almost non-investigated $[11,12]$. However, the revealing of such correlation (or its absence) would help to understand the mechanisms underlying the high level of the burden disease among students, assess possible tools for influence on the state of health through formation of health-saving behavior skills and, ultimately, on the efficiency of the educational process in the medical university. Based on these concepts, we have stated the goal of this study: to study the correlation between self-assessment of the life quality associated with health and widespread behavioral stereotypes in relation to health among students of medical universities - as social processes affecting the efficiency of the educational system.

\section{METHODOLOGICAL BASIS OF STUDY}

The methodological basis of study rests on the structural and functional approach which involves the study of functional dependencies between the social processes of implementation of behavioral stereotypes in relation to health by students of medical universities and their self-assessment of their physical and mental status. Now the structural-functional approach, originating with papers of T. Parsons and R. Merton [13,14], has been complemented and improved by their followers and is a valid and effective tool for the sociological analysis of adaptation and professional insocialization processes taking place in the educational system of the medical university, in particular. The specific scientific level of the functionalism methodology in this paper is presented by the attempt to discover functional relationships and their interpretation, the empirical level - by gathering and statistical processing the data array, correlation analysis of behavioral markers and indicators of self-assessment of the life quality associated with health.

\section{MATERIALS AND METHODS}

The data were collected in the period from 01.06.2019 to 01.03.2020 at the Pacific State Medical University (PSMU) and the Far Eastern Federal University (FEFU). The sample size was calculated taking into account the approximate variance of answers in previously carried out similar studies and represented 652 students of both sexes (324 (49.7\%) girls and 328 $(50.3 \%)$ boys), the average age of the participants was 20.3 years. The response rate was found to be $93.9 \%$.

Self-assessment of the life quality was carried out using the questionnaire "Brief form of self-assessment of the life quality related to health MOS SF-36" (MOS SF Medical Outcomes Study-Short Form) [15]. This questionnaire has been validated in Russian determining the constructive and discriminant validities. MOS SF-36 consists of two main domains and eight scales. Each scale is rated from 0 to 100 points.

When making the assessment of basic behavioral stereotypes in relation to health, we used the questionnaire of behavioral self-assessment of student of the medical university drawn up by authors based on the question list of Pozdeeda (2008) [15] taking into account the data obtained in studies of Bakaev, Bolotin, et al. [16]. Block A contains the initial and current attitudes in relation to health, including the attitude toward bad health habits; block B - the questions in regards to psychological comfort and psycho-hygiene, including items on interactions with parents, teachers, other students; block $\mathrm{C}$ - the questions on behavioral attitudes in accordance with the accommodation conditions and the educational system, including the eating behavior; block D - the questions on information awareness and cognitive capabilities; block E - on medical activity and committing to physical culture. Student made the assessments of each several questions in the range of 1 to 5 points (maximum positive answer).

When making statistical treatment of the material, the confidential interval of $\pm 5 \%$ was adopted, when comparing the data, the double-side analysis of variance (ANOVA) was carried out. The significance of correlation between variables was determined with a view to the Spearman's rho. The force of parameter association was rated as high within the range of coefficient values $\mathrm{R}=0.7-1.0$; mid-level $\mathrm{R}=0.4-0.6$.

\section{RESULTS}

In $27(4.1 \%)$ cases, the data of individual files were incomplete. This was associated either with errors in boxchecking or with unreadable corrections. The comparison of the number of questionnaires with complete and 
incomplete data according to Wald allowed us to reject hypothesis on statistical significance of non-available variables.

While analizing the results of study of the life quality associated with health, it was found that the highest point the students gave to the absence of somatic paid (SP85.6; reversible assessment), the lowest- to the emotional status (figure 1).

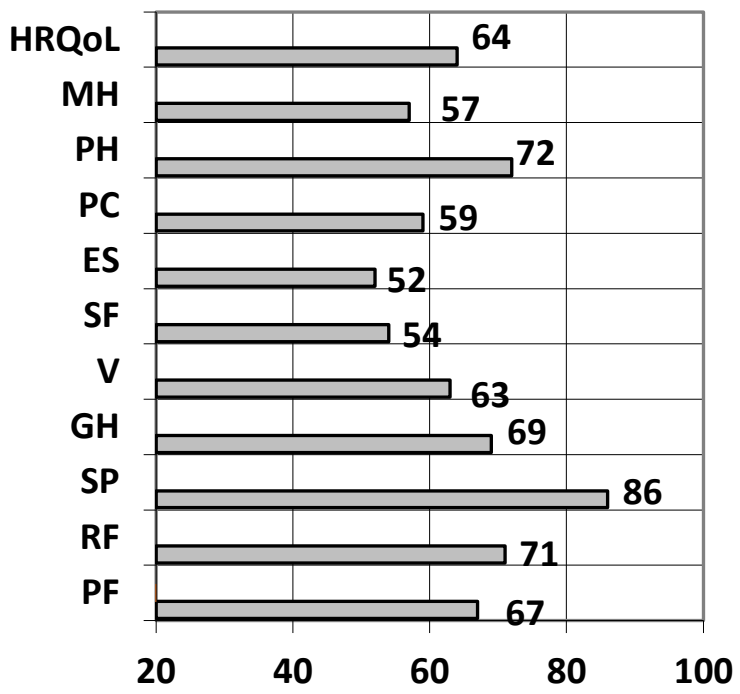

Figure 1 The self-assessment of health-related quality of life in medical students in MOS SF-36v2 scores $(n=652)$.

Note.

HRQoL - health-related quality of life

MH-mental health

$\mathrm{PH}$ - physical health

PC-psychological comfort

ES-emotional status

SF-social functioning

V- vitality

GH - General health

SP-somatic pain

RF-role-based functioning

$\mathrm{PF}$ - physical functioning

The mean assessment of the physical status was 72.0 points. The mental status assessment was far below- 56.8, mainly based on the variable values of the emotional status and social functioning. The composite assessment of the life quality concerned with health was 64.4 points, which corresponds to the regulatory value range.

The study of behavioral stereotypes in relation to health has allowed to establish that students gave a high rating to initial and current attitudes in relation to health (4.4 mean point). In particular, $86.3 \%$ denounce the availability of bad health habits. In Block B with the average grade of 3.9 point, attention is paid to the answers indicative of the high level of imitativeness significant to others when choosing the behavioral pattern and readiness to take as a pattern the behavioral make-up of friends $(67.3 \%)$, representatives of the teaching staff of the university $(23.5 \%)$, opinion leaders of the student community (21.1\%), "cyber" news-makers $(12.8 \%)$, parents $(11.4 \%)$ (more than 1 answer is allowed). The average value of answers in Block $\mathrm{C}$ (implemented attitudes on living and teaching conditions) was 3.5 points, Block D (information activity) - 4.0 point, Block E (medical activity, physical culture) - 3.2 point.

Figure 2 presents the correlation analysis findings of the correlation level between the total indicator of the life quality and variables quantitatively describing the behavioral activity in relation to health.

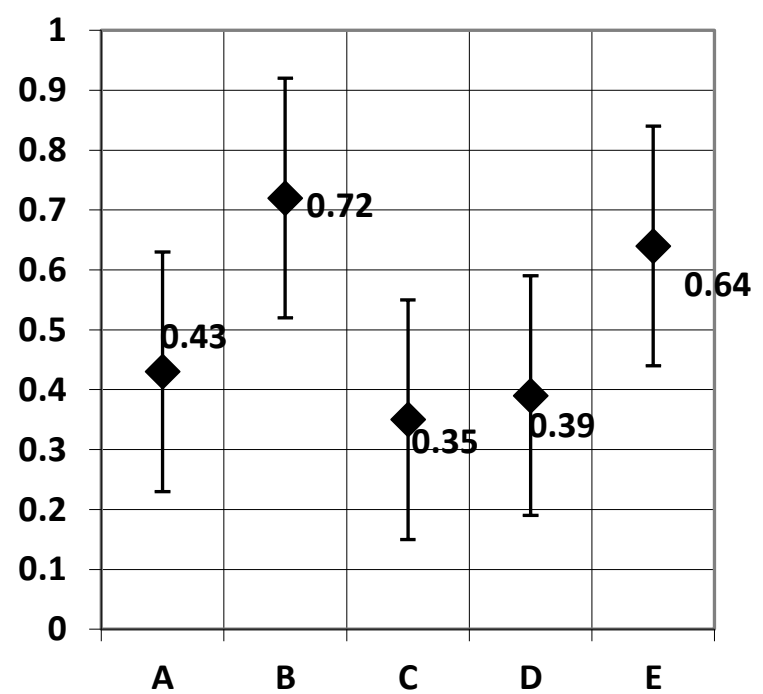

Figure 2 The correlation between the total variable of self-assessment of quality of life and the average values of the parameters of behavioral activity of medical students $(n=652)$.

A-attitudes towards health

B-interaction with significant others

$\mathrm{C}$-settings for training conditions

D-information activity

E-medical and physical activity

As you can see by submitted material, the strongest correlation of self-assessment of the life quality is noted with variables quantifying interaction with significant others $(0.72, \mathrm{p} \leq 0.05)$, as well as medical and physical activities $(0.64, \mathrm{p} \leq 0.05)$.

\section{DISCUSSION}

In empirical part of the study, it was found that students of medical professions assess their quality of life within reference values and physical status is reliably higher than psychological state. This is in agreement with the data of other authors carrying out the study of selfassessment of the life quality of university students $[7,8,15]$. Thus, in spite of the high incidence of disease confirmed in many modern researches, including our own $[11,12]$, students assess their physical abilities rather highly, which can be explained by significant inherent 
compensation and adaption abilities at $18-25$, as well as by significant load on the cognitive sphere of students from excessive teaching loads to excessive involvement in activity of online communities - which maybe abstract their minds from self-analysis, displace pathological signs to fringe of consciousness. Also students gave big points to the significance for making the behavioral solutions of initial and current attitudes in respect of health as well as information activity, which is typical and is along the lines of preceding observation over students of different professions.

From our point of view, the most interesting result from obtained ones is presented by the strong and medium degree of correlation of the life quality with variables quantifying interaction with significant others, as well as medical activity and attitude to physical loads. The structural and functional approach allows us to interpret these correlations as the influence of a wide range of processes and structures of the educational system on the life quality of students of medical professions. The functional dependence of the life quality on medical activity can be illustrated by attitudes towards the health-preserving behavior active in visiting a doctor at the appearance of symptoms of the disease, refusal of self-treatment, the use of contraceptives, etc. The functional influence on the life quality of physical activity is implemented in the large frequency and intensity of training loads, propensity to activities. At the same time, the possibility of developing the physical activity for every student is supported by the structure of the university educational system which has appropriate gyms equipped with exercise equipment, sports equipment, other sports accessories, swimming pools, etc.

Highly interesting is the found tendency to imitate the health-preserving behavior of significant others. Whereas the declared focus on the behavioral pattern of friends and opinion leaders of the student community is routine, then the imitation of representatives of the teaching staff looks rather unexpected. In principle, the imitation strategy of insocialization associated with adaptation in the educational system is relevant to views of G. Tarde [17] and his modern followers on the key importance of imitation and copying of behavioral attitudes for adaptation of a person to the new environmental conditions. Focus on behavioral stereotypes of significant others is in line with the concept of E. Thormdike [18] and researches who are in sync [19] about socially congruent behavior of a person in the new environment as a result of learning when choosing the most adaptive behavioral model allowing a person to integrate in new social interrelation with minimum of "damage". Such learning can take place according to A. Bandura [20] through "observation, imitation and identification".
Submitted results, to our opinion, can be useful to specialists in the field of medical and educational sociology, teachers, doctors-organizers, specialists of allied scientific disciplines.

\section{CONCLUSIONS}

1. In general, students of medical professions assess the life quality associated with health as satisfactory, however, they define the state of physical status as significantly better than mental. The level of emotional status received the lowest average self-assessment. At the same time, the respondents note the high degree of implementing the behavioral attitudes in relation to health (4.4 points), psycho-hygiene (3.9), somewhat less - in relation to informational, medical and physical activity. As significant others being examples for imitation in relation to health, students more often mention friends, representatives of teaching staff, opinion leaders of the student community.

2 . The variable quantifying the life quality associated with health has the strongest and medium correlation with indices characterizing interaction with significant others $(0.72, \mathrm{p} \leq 0.05)$, medical and physical activities $(0.64, \mathrm{p} \leq 0.05)$.

3. Analysis of behavioral stereotypes in relation to health and correlation of several of them with the life quality allows us to interpret professional insocialization of medical students within the structural and functional approach. The found functional correlations suggest that one of important adaptation mechanism of students in the educational system is imitation and copying significant others, focus on their behavioral stereotypes, with is in good agreement with views of G. Tarde, concept of A. Bandura on "observation, imitation and identification" and is consistent with the principle of E. Thorndike on choosing the most adaptive model of behavior.

\section{AUTHOR CONTRIBUTIONS}

Kosilov K.V. - conception, design, statistical treatment of material

Fedorishcheva E.K. - conception, design, writing the article, review of literature

Barabash O.A. - collection of material, statistical treatment of material, audit

Gareeva I.A. - conceptin, collection of material, selection of tools, literature review

\section{REFERENCES}

[1] Jochanan Benbassat Undesirable features of the medical learning environment: a narrative review of the literature. Adv Health Sci Educ Theory Pract 18(3) (2013) 527-36. DOI: https://doi.org/10.1007/s10459-012-9389-5 
[2] Blanka Klímová, Mobile Learning in Medical Edu cation, J Med Syst 42(10) (2018) 194. DOI: https://doi.org/10.1007/s10916-018-1056-9

[3] I.Yu. Melnikova, M.G Romantsov, Features of medical education and the role of a university teacher in the educational process at the present stage, International Journal of Experimental Education 11-2 (2013) 47-52. Retrieved from: http://www.expeducation.ru/ru/article/view?id=431 $\underline{4}$

[4] Lyakh Yulia Anatolyevna, Factors influencing the formation of the quality of education, Yaroslavl Pedagogical Bulletin 2 (2018) 8-16. DOI: https://doi.org/10.24411/1813-145X-2018-10001

[5] D.C. Jessop, M. Reid, L. Solomon, Financial concern predicts deteriorations in mental and physical health among university students, Psychol Health 35(2) (2020) 196-209. DOI: https://doi.org/10.1080/08870446.2019.1626393

[6] S.J. Slavin, Medical Student Mental Health: Culture, Environment, and the Need for Change, JAMA 316(21) (2016) 2195-2196. DOI: https://doi.org/10.1001/jama.2016.16396

[7] Julie Sigvartsen, Leiv Einar Gabrielsen, Eirik Abildsnes, Tonje H Stea, Christina Sandvand Omfjord, Gudrun Rohde. Exploring the relationship between physical activity, life goals and healthrelated quality of life among high school students: a cross-sectional study. BMC Public Health 15 (2016) 709. DOI: https://doi.org/10.1186/s12889-0163407-0.PMID:27488255

[8] X.Y. Wu, L.H. Han, J.H. Zhang, S. Luo, J.W. Hu, K. Sun, The influence of physical activity, sedentary behavior on health-related quality of life among the general population of children and adolescents: A systematic review, PLoS One 2017 Nov 9 12(11): e0187668.

DOI: https://doi.org/10.1371/journal.pone.0187668

[9] E.K. Fedorishcheva, I.A. Gareeva, K.V. Kosilov, Factors of influence on behavioral stereotypes in relation to health in medical students. Social and humanitarian knowledge Vol. 6 Iss. 3 (2020) 286299. DOI: https://doi.org/10.18255/2412-65192020-3-286-299

[10] E.K. Fedorishcheva, Comprehensive assessment of health-preserving behavior of medical students and directions for its optimization, Power and control in the East of Russia 3(88) (2019) 97-116. DOI https://doi.org/10.22394/1818-4049-2019-88-3-97$\underline{116}$
[11] V.V. Kuznetsov, R.A. Bayramov, E.A. Smirnov, E.K. Kosilova, K.V. Kosilov, Analysis of selfassessment of health and morbidity in senior students of medical and humanitarian specialties. Social aspects of population health 4(65) (2019) 16. DOI: https://doi.org/10.21045/2071-5021-2019$\underline{65-4-2}$

[12] V.V. Kuznetsov, R.A. Bayramov, E.A. Smirnov, E.K. Kosilova, K.V. Kosilov, Correlation between self-assessment of health status and the level of morbidity with academic performance in senior students of medical specialties, taking into account the influence of socio-economic and demographic characteristics. Medical miscellany 5-6(61) (2019) 10-15. DOI: https://doi.org/10.21145/2499-99542019-5-10-15

[13] R.K. Merton, Social theory and social structure, Sociological research 2 (1992) 118-124.

[14] R.K. Merton, Social theory and social structure, Sociological research 3 (1992) 104-114.

[15] J. Brazier, J. Roberts, M. Deverill, The estimation of a preference-based measure of health from the SF36, Journal of Health Economics 21 (2002) 271-292. DOI: https://doi.org/10.1016/s01676296(01)00130-8

[15] Fedorishcheva E.K. Comprehensive assessment of health-preserving behavior of medical students and directions for its optimization. Power and control in the East of Russia 3(88) (2019) 97-116. DOI: https://doi.org/10.22394/1818-4049-2019-88-3-97$\underline{116}$

[16] V.V. Bakaev, A.E. Bolotin, Indicators of students' readiness for health-preserving behavior, Scientific notes of the University named P.F. Lesgaft 12(106) (2013) 36-39. DOI: https://doi.org/10.34835

[17] Tarde, Gabriel de, The laws of imitation, - Moscow: Academic project, 2011, 302 p.

[18] E. Thorndike, J.B. Watson, Behaviorism, Principles of teaching based on psychology, Psychology as a science of behaviour, M.: AST-LTD, 1998, 704 p.

[19] E.I. Rasskazova, T.Yu Ivanova, Motivational health-related behaviors: problem of the "gap" between intention and action, Psychology. Journal of the Higher School of Economics Vol. 12. Iss. 1 (2015) 105-130. Retrieved from: https://psyjournal.hse.ru/2015-12-1/148010788.html

[20] A. Bandura, Social learning theory, - SPb.: Eurasia, 2000, 320 p. 